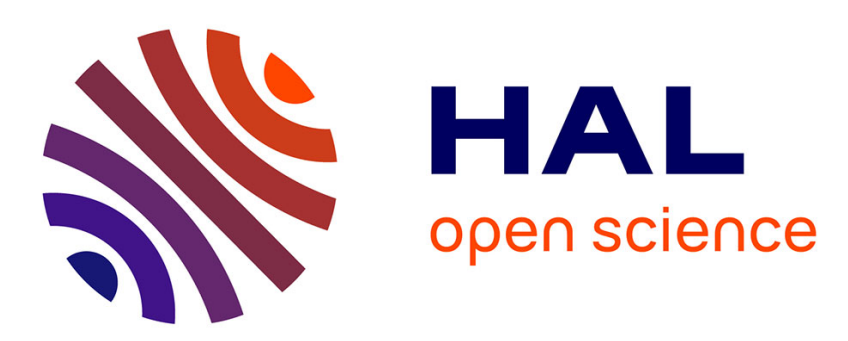

\title{
Maladie hypertensive des personnes nées en Afrique subsaharienne adressées à un service spécialisé : étude transversale comparative
}

\author{
Thierry Gombet, Olivier Steichen, Pierre-Francois Plouin
}

\section{- To cite this version:}

Thierry Gombet, Olivier Steichen, Pierre-Francois Plouin. Maladie hypertensive des personnes nées en Afrique subsaharienne adressées à un service spécialisé : étude transversale comparative. Bulletin de l'Académie Nationale de Médecine, 2007, 191 (8), pp.1745-1755. hal-01171836

\section{HAL Id: hal-01171836 \\ https://hal.sorbonne-universite.fr/hal-01171836}

Submitted on 8 Jul 2015

HAL is a multi-disciplinary open access archive for the deposit and dissemination of scientific research documents, whether they are published or not. The documents may come from teaching and research institutions in France or abroad, or from public or private research centers.
L'archive ouverte pluridisciplinaire HAL, est destinée au dépôt et à la diffusion de documents scientifiques de niveau recherche, publiés ou non, émanant des établissements d'enseignement et de recherche français ou étrangers, des laboratoires publics ou privés. 


\section{Maladie hypertensive des sujets nés en Afrique subsaharienne adressés à un service spécialisé : étude transversale comparative}

MOTS-ClES : ACCIDENT VASCULAIRE CEREBRAL. GROUPES ETHNIQUES. HYPERALDOSTERONISME. HYPERTENSION. HYPERTENSION, RENOVASCULAIRE. PROTEINURIE

Hypertensive disease in subjects born in sub-Saharan Africa or in Europe referred to a hypertension unit: a cross-sectional study

KEY-WORDS: CEREBROVASCULAR ACCIDENT. ETHNIC GROUPS. HYPERALDOSTERONISM. HYPERTENSION. HYPERTENSION, RENOVASCULAR. PROTEINURIA

Thierry GOMBET*, Olivier STEICHEN**, Pierre-François PLOUIN ${ }^{*}$

\section{RESUME}

L'hypertension des sujets noirs est réputée plus grave que celle des sujets blancs, mais la plupart des données proviennent d'études nord-américaines. Nous avons comparé les caractéristiques de l'hypertension et la prévalence des facteurs de risque associés, des complications cardiovasculaires et des causes d'hypertension secondaire chez 539 hypertendus nés en Afrique subsaharienne et chez 2610 hypertendus nés en Europe appariés sur l'âge et le sexe. Par rapport aux hypertendues européennes, les hypertendues africaines étaient plus corpulentes (indice de masse corporelle $\left.28,8 v s 26,3 \mathrm{~kg} / \mathrm{m}^{2}, p<0,001\right)$ et plus souvent diabétiques (12 vs 5\%, $p<0,001)$. Les hypertendus Africains des deux sexes avaient une pression artérielle systolique plus élevée (152 vs $148 \mathrm{mmHg}, p<0,001)$, plus souvent un antécédent d'accident vasculaire cérébral (11,7 vs 6,7\%, $p<0,001)$, plus souvent une protéinurie à la bandelette (32 vs 18\%, $p<0,001$ ), et moins souvent une exposition au tabac ou une hyperlipidémie que leurs témoins européens. Les hypertendus Africains étaient plus souvent sous traitement lors de leur première consultation que leurs témoins européens (84vs 74\%, p<0,001), avec un traitement comportant plus souvent un diurétique (54 vs 46\%, $p=0,001$ ) et un inhibiteur calcique (49vs 58\%, $p=0,001$ ). La proportion d'hypertensions secondaires était similaire dans les 2 groupes mais les hypertendus Africains avaient plus souvent un hyperaldostéronisme primaire $(12 \mathrm{vs} 7 \%, p=0,001)$ et moins souvent une maladie rénovasculaire (1 vs 5\%, p=0,001) que leurs témoins européens. En conclusion, le retentissement cérébrovasculaire et rénal plus sévère chez les patients d'origine africaine n'est pas complètement expliqué par les différences observées dans la systolique et les cofacteurs de risque. La distribution des hypertensions secondaires chez les sujets africains mérite une étude spécifique.

\section{SUMMARY}

Hypertensive disease is reported to be more severe in black patients than in white patients, but most available data concern African-Americans. We compared blood pressure history and levels, the prevalence of associated risk factors, renal and cardiovascular complications, and secondary forms of hypertension in patients born in sub-Saharan Africa and in up to five patients born in Europe matched for age and sex. Compared to European hypertensive women, African hypertensive women were more corpulent (body-mass index $28.8 \mathrm{vs.} 26.3 \mathrm{~kg} / \mathrm{m}^{2}$, $p<0.001)$ and more often diabetic (12 vs. 5\%, p<0.001). Hypertensive men and women born in sub-Saharan Africa had higher systolic blood pressure (152 vs. $148 \mathrm{mmHg}, p<0.001)$, were more likely to have a history of stroke (11.7 vs. 6.7\%, $p<0.001)$ and less likely to have a history of smoking or hyperlipidemia than paired controls born in Europe. Patients born in sub-Saharan Africa more frequently had proteinuria at dipstick (32 vs. $18 \%, p<0.001$ ), irrespective of blood pressure levels and the presence of diabetes, than their paired European controls. The proportion of patients taking antihypertensive drugs at referral, the distribution of

*AP-HP, Hôpital Européen Georges Pompidou, unité d'hypertension artérielle, 20 rue Leblanc, 75908 Paris cedex 15 ; Université Paris Descartes, Faculté de Médecine, Paris.

***AP-HP, Hôpital Tenon, service de médecine interne, 4 rue de la Chine, 75020 Paris ; Université Pierre et Marie Curie-Paris6, Faculté de Médecine, Paris.

Tirés-à-part: Professeur Pierre-François Plouin, unité d'Hypertension artérielle, Hôpital Européen Georges Pompidou, 75908 Paris cedex 15 
antihypertensive agents, and the proportion of patients having a complementary work-up after referral did not differ between patients born in sub-Saharan or in Europe. Complementary work-up disclosed a similar overall prevalence of secondary hypertension in the two geographic groups. However, patients born in sub-Saharan Africa were more likely to have primary hyperaldosteronism (12 vs. 7\%, p=0.001) and less likely to have renovascular disease ( 1 vs. 5\%, $p=0.001)$ than European controls. In conclusion, the higher prevalence of cardiovascular and renal complications in patients born in Sub-Saharan than in patients born in Europe seems not to be solely explained by the observed differences in blood pressure levels or associated risk factors at referral. The difference in the distribution of secondary forms of hypertension requires further study.

\section{INTRODUCTION}

L'hypertension artérielle (HTA) est en Afrique subsaharienne (AS) un problème d'une immense portée médicale et économique du fait de sa grande prévalence en milieu urbain, de son dépistage tardif ou incomplet, des problèmes économiques liés à sa prise en charge, et des conséquences de ses complications [1,2]. Les personnes nées en AS qui vivent en France et celles qui vivent dans leur pays natal ont en commun des caractéristiques culturelles qui incluent la langue, la religion, les habitudes alimentaires et l'ascendance. Celles qui vivent en France sont néanmoins exposées à un environnement différent; elles partagent l'environnement climatique, et en partie l'environnement alimentaire et socio-économique des personnes nées en Europe.

En matière d'HTA, les similitudes et différences entre hypertendus africains-américains et américains d'origine européenne sont assez bien connues [3,4], de même que les différences entre hypertendus nés aux Antilles, généralement de lointaine ascendance africaine, et les hypertendus nés en France métropolitaine, généralement d'ascendance européenne [5]. En revanche, on connaît peu chez les personnes résidant en France les similitudes et différences entre les hypertendus nés en AS et les hypertendus nés en Europe. L'étude de ces similitudes et différences devrait reposer sur la comparaison de la prévalence de l'HTA et de ses complications dans des échantillons représentatifs des populations concernées. Il est malheureusement difficile d'obtenir un échantillon représentatif d'une population récemment immigrée comme celle des personnes nées en AS et résidant en France. La question peut être approchée par l'analyse d'une population hospitalière. Nous avons comparé les caractéristiques de l'HTA et la prévalence des facteurs de risque associés, des complications cardiovasculaires et des causes d'HTA secondaire chez des hypertendus nés en AS et des hypertendus nés en Europe adressés à un service spécialisé.

\section{PATIENTS ET METHODES}

\section{Sélection des patients et extraction des données.}

Nous avons inclus les patients hypertendus consultant pour la première fois entre 2001 et 2006 dans notre unité d'hypertension, nés en Europe ou dans les pays suivants d'AS, classés par effectifs décroissants de patients inclus : Congo, Cameroun, Cote d'Ivoire, Sénégal, Mali, Togo, Bénin, Madagascar, Guinée-Bissau, Guinée-Conakry, Mauritanie, Angola, Gabon, Burkina Faso, République Centre-Africaine, Soudan, Ghana, Niger, Tchad, Zambie, Nigeria, Ouganda, Ethiopie et Gambie. Les patients dont la pression artérielle (PA) était normale en l'absence de traitement antihypertenseur, les patients français nés dans un département ou territoire d'outre-mer et les patients dont la première visite avait directement lieu en hôpital de jour ou de semaine ont été exclus. 
L'observation médicale de l'unité d'HTA est informatisée depuis 1975 ; les données cliniques et paracliniques des patients sont enregistrées dans une base de données hospitalières [6]. Il est ainsi possible d'accéder à travers des requêtes informatiques aux principaux éléments de l'histoire médicale, au traitement en cours lors de la visite, à l'évaluation du contrôle de l'HTA et des cofacteurs de risque cardiovasculaire, et enfin à l'évaluation de la cause de l'HTA et de son retentissement sur les organes cibles.

La valeur des variables suivantes au moment de la première consultation a été extraite : âge, sexe, tabagisme actuel et/ou passé ; ancienneté connue de l'HTA; antécédents connus de dyslipidémie, de diabète, d'insuffisance rénale, d'accident vasculaire cérébral (AVC), de cardiopathie ischémique ou d'insuffisance cardiaque; indice de masse corporelle (IMC: poids/taille ${ }^{2}$ ), pression artérielle (PA), traitement en cours ; résultats de l'électrocardiogramme (indice de Sokolow: R1 + S5 ou S6; indice de Cornell : RL + S3); et présence d'une protéinurie à la bandelette (à partir d'une croix). La PA était mesurée par une infirmière entraînée utilisant un moniteur semi-automatique validé (Omron 705CP). Trois mesures de PA ont été obtenues en position assise après 5 minutes de repos, et la moyenne de la deuxième et de la troisième mesures retenue pour définir la PA clinique, comme décrit précédemment [7]. Chez les patients recevant un traitement antihypertenseur, le score de traitement était défini par le nombre de classes thérapeutiques prescrites parmi les suivantes : diurétiques, bétabloquants, antagonistes calciques, inhibiteurs de l'enzyme de conversion de l'angiotensine, antagonistes du récepteur $\mathrm{AT}_{1}$ de l'angiotensine II et antihypertenseurs d'action centrale.

Chez les patients ayant eu une exploration complémentaire en hôpital de jour ou de semaine, on a également recueilli la cholestérolémie, la glycémie à jeun, la créatininémie, l'index de masse ventriculaire gauche (masse ventriculaire gauche estimée en échographie rapportée à la surface corporelle ou à la taille ${ }^{2,7}$, et le résultat de l'enquête étiologique. Une éventuelle étiologie rénale parenchymateuse, rénovasculaire ou surrénale a été retenue si elle apparaissait dans le compte rendu informatisé de sortie après une hospitalisation. Les méthodes pour le dépistage et la confirmation de ces étiologies sont décrites par ailleurs $[8,9]$.

\section{Analyse statistique.}

Chaque patient né en AS a été comparé à 5 patients nés en Europe appariés sur l'âge ( \pm 1 an) et le sexe, afin de s'affranchir de l'influence majeure de ces deux variables sur la maladie hypertensive et les cofacteurs de risque cardiovasculaire. Les données sont présentées en nombre de patients (pourcentage) pour les variables binaires et en moyenne ( \pm écart-type) pour les variables quantitatives. Pour chaque variable, le nombre de patients avec des données disponibles est indiqué. Les comparaisons appariées entre hypertendus nés en AS et hypertendus nés en Europe ont été effectuées en régression logistique conditionnelle univariée. Une interaction avec le sexe a été recherchée de façon systématique dans toutes les comparaisons; le cas échéant, les résultats pour les femmes et pour les hommes sont présentés séparément. Pour ces analyses univariées exploratoires, le seuil de significativité retenu après correction de Bonferroni était $\alpha=0,05 / 50=0,001$. En effet, des comparaisons indépendantes étaient prévues sur 32 variables, avec une duplication en cas d'hétérogénéité entre hommes et femmes, soit une cinquantaine de comparaisons. Lorsque des différences étaient observées entre hypertendus africains et européens en analyse univariée, l'effet des facteurs de confusion potentiels a été étudié par ajustement en régression logistique 
conditionnelle multivariée. Le seuil de significativité retenu était alors $\alpha=0,05$. Les analyses statistiques ont été réalisées dans Stata 8.2 (StataCorp, College Station, Texas, États-Unis).

\section{RESULTATS}

\section{Données de la première consultation.}

Les caractéristiques initiales des patients originaires d'AS et d'Europe sont comparées dans le Tableau 1. Les hypertendus nés en AS avaient une plus grande prévalence d'AVC et une plus faible prévalence d'hyperlipidémie et d'exposition au tabac que les hypertendus nés en Europe. Leur PA et leurs indices électrocardiographiques étaient plus élevés que ceux des hypertendus nés en Europe et ils avaient plus souvent une protéinurie à la bandelette. La différence de prévalence des AVC restait significative après ajustement sur la PA systolique et les cofacteurs de risque dont l'IMC $(\mathrm{p}=0,001)$. La différence de protéinurie à la bandelette restait significative après ajustement sur l'IMC, le diabète et la PA systolique $(p<0,001)$. La différence de PA entre hypertendus nés en AS et en Europe restait significative après ajustement sur l'IMC et le diabète ( $p=0,003$ pour la systolique ; $p=0,001$ pour la diastolique). Les femmes nées en AS étaient plus corpulentes et plus souvent diabétiques que les femmes nées en Europe et cette dernière différence restait significative après ajustement sur l'IMC $(\mathrm{p}=0,004)$.

Les hypertendus nés en AS étaient plus souvent traités et avaient un score de traitement plus élevé que leurs pairs nés en Europe. La plus grande fréquence du traitement antihypertenseur à la première consultation restait significative $(\mathrm{p}=0,007)$ après ajustement sur la $\mathrm{PA}$ et les comorbidités (diabète, AVC, cardiopathie ischémique, insuffisance cardiaque, insuffisance rénale). Ils recevaient plus souvent un diurétique et un inhibiteur calcique que les hypertendus originaires d'Europe et cette différence restait significative après ajustement sur le score de traitement ( $\mathrm{p}=0,01$ et $\mathrm{p}=0,007$ respectivement).

\section{Exploration complémentaire.}

La fréquence et les résultats de l'exploration complémentaire des patients originaires d'AS et d'Europe sont comparées dans le Tableau 2. La fréquence plus importante des consultations et hospitalisations dans les 6 mois chez les patients originaires d'AS était associée à la plus grande sévérité de la maladie hypertensive évaluée lors de la consultation initiale (respectivement $\mathrm{p}=0,98$ et $\mathrm{p}=0,74$ pour les consultations et les hospitalisations après ajustement sur la PA systolique, le diabète et le retentissement sur les organes cibles : AVC, insuffisance cardiaque, cardiopathie ischémique et protéinurie à la bandelette).

Les patients africains avaient des indices de masse ventriculaire gauche plus importants que leurs pairs européens, qu'ils soient indexés sur la surface corporelle ou sur la taille à la puissance $^{2,7}$. Le plus bas niveau de cholestérolémie chez les patients nés en AS n'était pas uniquement expliqué par la plus faible prévalence des dyslipidémies $(p<0,001$ après ajustement sur ce facteur). En revanche, le plus haut niveau de glycémie chez ces patients était expliqué par la plus grande prévalence du diabète et du surpoids ( $\mathrm{p}=0,17$ après ajustement sur ces facteurs). La créatininémie des patients africains était plus élevée que celle des patients européens. 


\section{COMMENTAIRES}

Les principaux constats tirés de cette étude sont la plus grande fréquence des antécédents cardiovasculaires; le plus haut niveau de la PA à la première consultation; et le retentissement cardio-rénal plus sévère de l'HTA chez les hypertendus nés en AS que chez leurs pairs nés en Europe. Ces différences ne sont pas inattendues [1-4,10,11]. Bien que la distribution des cofacteurs de risque (corpulence, tabagisme, diabète, dysplipidémie) soit différente dans les deux groupes, elle ne semble pas suffire à expliquer la différence de sévérité de la maladie hypertensive.

Nos résultats ne paraissent pas non plus directement expliqués par une différence dans l'accès aux soins [12]. Les patients nés en AS avaient à la première consultation une PA plus élevée et un niveau maximum connu de PA systolique plus élevé - que leurs pairs nés en Europe. Néanmoins leur HTA n'était pas plus ancienne et elle était plus souvent traitée que celle de leurs pairs. Les traitements prescrits - plus nombreux comme le justifiait leur niveau de PA utilisaient plus souvent les diurétiques et les inhibiteurs calciques, des agents bien adaptés à l'HTA des sujets d'ascendance africaine $[4,12,13]$. Ces patients nés en AS ont été plus souvent explorés en hospitalisation et suivis en consultation que leurs pairs, à proportion de leur HTA plus sévère. Au sein de cette population référée par les correspondants d'une unité spécialisée d'HTA, il n'y avait pas donc de biais évident pouvant suggérer une moins bonne prise en charge des patients nés en AS. Néanmoins les complications et les atteintes précliniques cardiovasculaires et rénales étaient plus fréquentes chez ces patients que chez leurs pairs européens, et cette différence de fréquence persistait après ajustement sur la PA.

Ces données suggèrent que le facteur hémodynamique - le niveau de la $\mathrm{PA}$ - n'est pas la seule explication de la plus grande prévalence des AVC, de l'hypertrophie ventriculaire gauche ou de l'atteinte rénale chez les sujets nés en AS. Des différences dans le bilan sodé et son contrôle neurohormonal, d'origine environnementale ou génétique, peuvent rendre compte de complications cardiovasculaires et rénales plus fréquentes à niveau égal de PA $[14,15]$. Notre étude n'avait ni les moyens ni l'objectif d'une étude physiopathologique des cas d'HTA essentielle qui formaient la majorité du recrutement. Toutefois des différences étaient présentes parmi les étiologies d'HTA secondaires que nous avons détectées. La maladie rénovasculaire, localisation de l'athérosclérose dans la circulation rénale, était moins fréquente chez les sujets originaires d'AS que chez leurs pairs d'Europe, ce qui peut refléter la moindre prévalence chez les premiers de l'hypercholestérolémie et de l'exposition au tabac. $\mathrm{Au}$ contraire l'hyperaldostéronisme primaire semblait plus fréquent chez les sujets nés en AS. Cette forme d'HTA est connue pour entraîner, à niveau égal de PA, des conséquences plus sévères en termes de morbidité cérébrovasculaire, d'hypertrophie ventriculaire gauche et de protéinurie $[16,17]$.

Cette étude a plusieurs limites. Elle est rétrospective ; même si le recueil des données a été prospectif, utilisant une base de données standardisée, le recueil des données n'est pas exhaustif. Certaines variables intéressantes n'ont pas pu être étudiées de façon systématique comme le niveau d'éducation, le niveau de prise en charge médicale, la natriurèse et la créatininurie. Elle porte sur une population hospitalière, nécessairement biaisée : de nombreux hypertendus nés en AS et vivant en France ne sont pas référés dans un centre d'HTA, et il en est de même pour de nombreux hypertendus nés en Europe. Il serait d'ailleurs difficile de constituer un échantillon représentatif des hypertendus nés en AS et résidant en France parce qu'il s'agit d'une population migrante et parce que les pays d'origine sont nombreux. Enfin 
l'ajustement statistique sur le niveau de la PA n'apporte pas une certitude sur le poids relatif du niveau de la PA et de facteurs non hémodynamiques dans le retentissement cardiaque, cérébral et rénal de l'HTA.

Notre étude a cependant des implications pratiques. La prise en charge des hypertendus nés en AS devrait être plus précoce qu'elle ne l'est actuellement. Un contrôle strict de la PA devrait être doublé d'objectifs non tensionnels : contrôler les facteurs de risque associés et réduire la microalbuminurie si elle est présente $[4,18]$. Les différences liées au sexe concernant l'IMC et la prévalence du diabète indiquent qu'une prise en charge diététique est importante chez les femmes nées en AS. Enfin une recherche spécifique devrait être consacrée au dépistage et à l'analyse des HTA secondaires chez les patients nés en AS.

\section{Remerciements}

Le docteur Thierry Gombet a bénéficié pour ce travail d'une bourse de l'Académie Nationale de Médecine 


\section{Bibliographie}

[1] COOPER R.S., ROTIMI C.N., KAUFMAN J.S., MUNA W.F., MENSAH G.A. - Hypertension treatment and control in sub-Saharan Africa: the epidemiological basis for policy. Br. Med. J., $1998,316,614-617$

[2] OPIE L.H., SEEDAT Y.K. - Hypertension in sub-Saharan African populations. Circulation, $2005,112,3562-3568$.

[3] COOPER R., ROTIMI C. - Hypertension in blacks. Am. J. Hypertens., 1997, 10, 804-812.

[4] DOUGLAS J.G., BAKRIS G.L., EPSTEIN M. et al. - Management of high blood pressure in African Americans: consensus statement of the Hypertension in African Americans Working Group of the International Society on Hypertension in Blacks. Arch. Intern. Med., 2003, 163, 525-541.

[5] INAMO J., LANG T., ATALLAH A. et al. - Prevalence and therapeutic control of hypertension in French Caribbean regions. J. Hypertens., 2005, 23, 1341-1346.

[6] Degoulet P., Chatellier G., Devriès C., Lavril M., Ménard J. - Computer-assisted techniques for evaluation and treatment of hypertensive patients. Am. J. Hypertens., 1990, 3, 156-63.

[7] LA BATIDE-ALANORE A., CHATELliER G., BOBRIE G., FOFOL I., PLOUIN P.F. Comparison of nurse- and physician-determined clinic blood pressure levels in patients referred to a hypertension clinic: implications for subsequent management. J. Hypertens., 2000, 18, 391398.

[8] PLOUIN P.F., LA BATIDE ALANORE A., FIQUET-KEMPF B. et al - Hypertensions secondaires à des causes curables chez l'adulte. Presse Med. 2002, 31, 371-378.

[9] PLOUIN P.F., BOBRIE G., CHATELLIER G. - How to manage hypertension in clinical practice, and what can be expected from a hypertension clinic. In Hypertension, A Zanchetti ed. (McGraw-Hill Clinical Medicine Series), 2001, pp. 241-251.

[10] CRUICKSHANK J.K., MBANYA J.C., WILKS R. et al. - Hypertension in four African-origin populations: current 'Rule of Halves', quality of blood pressure control and attributable risk of cardiovascular disease. J. Hypertens., 2001, 19, 41-46.

[11] ATALLAH A., INAMO J., LANG T. et al. - Une bonne couverture sociale et un bon accès au soin : un prérequis nécessaire mais insuffisant pour faire disparaître une disparité de prise en charge de l'HTA selon le niveau socio-économique. Arch. Mal. Cour Vaiss., 2006, 99, 674-678.

[12] SARELI P., RADEVSKI I.V., VALTCHANOVA Z.P. et al. - Efficacy of different drug classes used to initiate antihypertensive treatment in black subjects: results of a randomized trial in Johannesburg, South Africa. Arch. Intern. Med., 2001, 161, 965-971.

[13] WRIGHT J.T. Jr, DUNN J.K., CUTLER J.A. et al. - Outcomes in hypertensive black and nonblack patients treated with chlorthalidone, amlodipine, and lisinopril. JAMA., 2005, 293, 1595-1608.

[14] DU CAILAR G., RIBSTEIN J., MIMRAN A. - Dietary sodium and target organ damage in essential hypertension. Am. J. Hypertens., 2002, 15, 222-229.

[15] SCHMIDT B.M., SCHMIEDER R.E. - Aldosterone-induced cardiac damage: focus on blood pressure independent effects. Am. J. Hypertens., 2003, 16, 80-86. 
[16] MILLIEZ P., GIRERD X., PLOUIN P.F. et al. Evidence for an increased rate of cardiovascular events in patients with primary aldosteronism. J. Am. Coll. Cardiol., 2005, 45, 1243-1248.

[17] ROSSI G.P., BERNINI G., DESIDERI G. et al. Renal damage in primary aldosteronism: results of the PAPY Study. Hypertension, 2006, 48, 232-238.

[18] ASSELBERGS F.W., HILLEGE H.L., VAN GILST W.H. - Framingham score and microalbuminuria: combined future targets for primary prevention? Kidney Int. Suppl., 2004, 92, S111-114. 
Tableau 1 : Caractéristiques initiales des patients nés en Europe ou en Afrique sub-saharienne

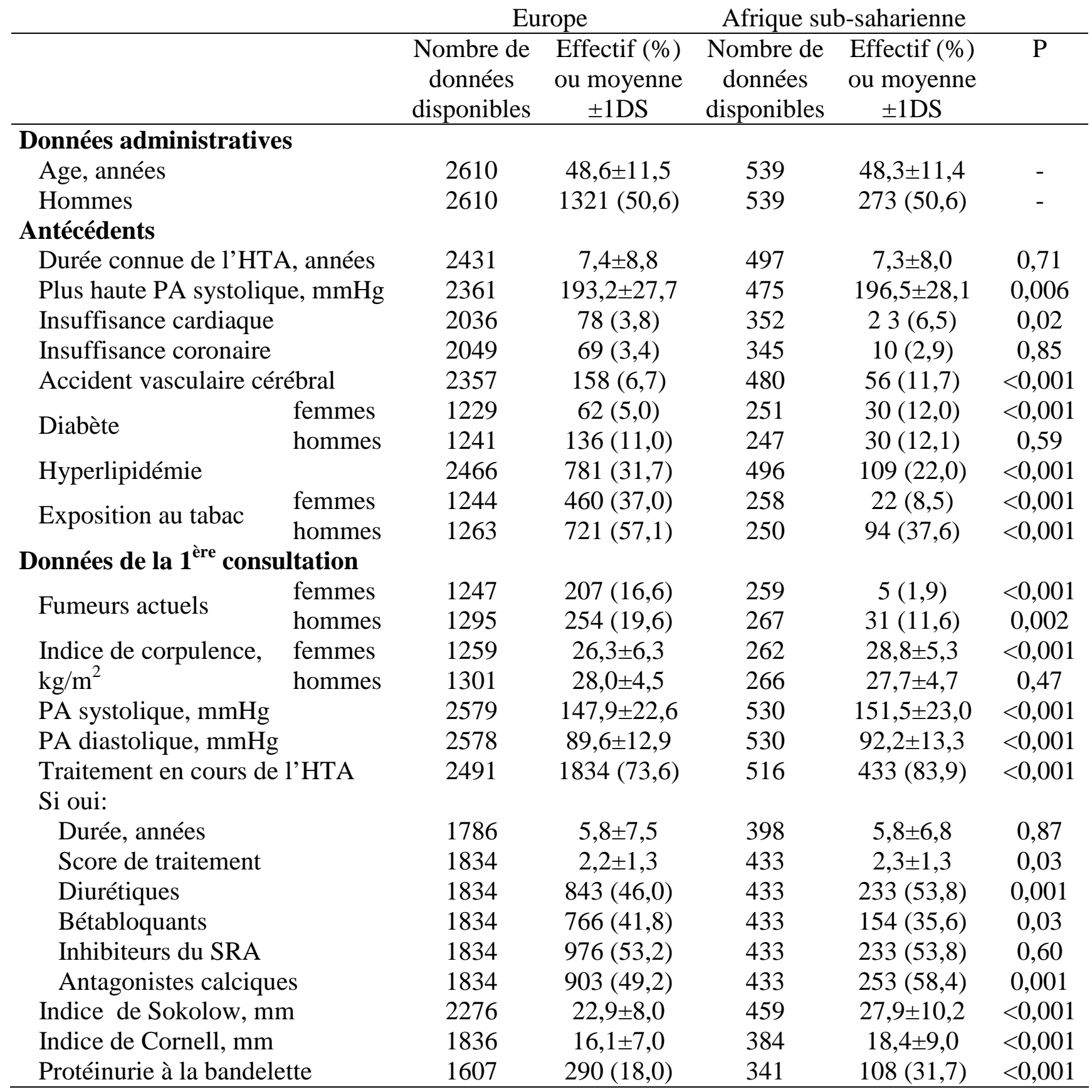

HTA: hypertension; PA: pression artérielle; SRA: système rénine-angiotensine 
Tableau 2 : Exploration complémentaire des patients nés en Europe ou en Afrique sub-saharienne

\begin{tabular}{|c|c|c|c|c|c|}
\hline & \multicolumn{2}{|c|}{ Europe } & \multicolumn{2}{|c|}{ Afrique sub-saharienne } & \multirow[b]{2}{*}{$\mathrm{P}$} \\
\hline & $\begin{array}{c}\text { Nombre de } \\
\text { données } \\
\text { disponibles } \\
\end{array}$ & $\begin{array}{l}\text { Effectif (\%) } \\
\text { ou moyenne } \\
\quad \pm 1 \mathrm{DS}\end{array}$ & $\begin{array}{c}\text { Nombre de } \\
\text { données } \\
\text { disponibles } \\
\end{array}$ & $\begin{array}{l}\text { Effectif }(\%) \\
\text { ou moyenne } \\
\pm 1 \mathrm{DS} \\
\end{array}$ & \\
\hline $\begin{array}{l}\text { Patients ayant eu } \geq 1 \text { consultation dans les } 6 \\
\text { mois suivant la } 11^{\text {ere }} \text { consultation }\end{array}$ & 2610 & $1309(50,2)$ & 539 & $318(59,0)$ & $<0,001$ \\
\hline $\begin{array}{l}\text { Patients ayant eu } \geq 1 \text { hospitalisation dans les } \\
6 \text { mois suivant la } 1^{\text {ère }} \text { consultation }\end{array}$ & 2610 & $1310(50,2)$ & 539 & $314(58,3)$ & 0,001 \\
\hline Cholestérolémie, mmol/l & 2124 & $5,3 \pm 1,1$ & 449 & $5,0 \pm 1,1$ & $<0,001$ \\
\hline Glycémie mmol/ & 938 & $5,4 \pm 1,1$ & 214 & $5,7 \pm 1,9$ & $<0,001$ \\
\hline Glycemie, mmol/1 & 996 & $5,9 \pm 1,5$ & 210 & $5,9 \pm 1,7$ & 0,823 \\
\hline Créatininémie, $\mu$ mol/1 & 2230 & $85,0 \pm 38,6$ & 480 & $98,8 \pm 66,4$ & $<0,001$ \\
\hline Index de masse ventriculaire gauche, $\mathrm{g} / \mathrm{m}^{2}$ & 888 & $96,5 \pm 30,5$ & 239 & $99,9 \pm 33,3$ & 0,02 \\
\hline Index de masse ventriculaire gauche, $\mathrm{g} / \mathrm{m}^{2,7}$ & 889 & $44,5 \pm 14,9$ & 240 & $47,3 \pm 16,6$ & 0,002 \\
\hline HTA secondaire documentée & 2196 & $401(18,3)$ & 440 & $89(20,2)$ & 0,43 \\
\hline Hyperaldostéronisme primaire & 2196 & $155(7,1)$ & 440 & $54(12,3)$ & 0,001 \\
\hline Néphropathie parenchymateuse & 2196 & $81(3,7)$ & 440 & $27(6,1)$ & 0,03 \\
\hline Maladie rénovasculaire & 2196 & $104(4,7)$ & 440 & $6(1,4)$ & 0,001 \\
\hline
\end{tabular}

HTA: hypertension 